\title{
When an academic culture based on self-enhancement values undermines female students' sense of belonging, self-efficacy, and academic choices
}

Cristina Aelenei ${ }^{1}$, Delphine Martinot ${ }^{2}$, Alyson Sicard $^{2}, \&$ Céline Darnon ${ }^{2}$

\section{Affiliations:}

1: Laboratoire de Psychologie Sociale: contextes et régulation (LPS) - EA 4471 - Université Paris Descartes, Paris, France

2: Laboratoire de Psychologie Sociale et Cognitive (LAPSCO), CNRS UMR 6024, Université Clermont Auvergne, Clermont-Ferrand, France 


\begin{abstract}
Although overall women are better represented in higher education than men, women's psychological experience in various academic contexts is qualified by a decreased sense of belonging and academic self-efficacy, including in fields where they are not targeted by a negative stereotype. To clarify this phenomenon, we develop the hypothesis of a mismatch between female students' values and the values associated with success in the increasingly selective realm of higher education. We argue that, whatever the fields of study, these values are self-enhancement values (competitiveness, self-affirmation, dominance). Three studies showed that when success was depicted in terms of self-enhancement values, women-but not men-expressed a lower sense of belonging, had lower self-efficacy and were less likely to pursue a given academic opportunity both in STEM and non-STEM fields of study. These effects did not appear in an academic context depicting success as being rooted in selftranscendence values (helpfulness, cooperation, benevolence).
\end{abstract}

Keywords: gendered values, higher education, sense of belonging, academic self-efficacy, academic choices 
When an academic culture based on self-enhancement values undermines female students' sense of belonging, self-efficacy, and academic choices

The higher education system in the western countries is becoming increasingly selective (Darnon, Dompnier, Delmas, Pulfrey, \& Butera, 2009; Jury, Darnon, Dompnier, \& Butera, 2016), creating a negative social interdependence dynamic and thus, an academic culture emphasizing self-enhancement values (e.g., competiveness, self-affirmation, dominance, Schwartz, 2012). We argue that depicting academic success as being linked to these values may have a deleterious effect on women's academic experience. We lay the ground for this proposal by looking first at more nuanced indicators on gender inequalities in the western higher education system, and at how past research may be limited in fully addressing these dynamics.

\section{Gender in Higher Education: From general tendencies to more nuanced}

\section{indicators}

Women today represent the majority of students in higher education in the western countries (Fiske \& UNESCO, 2012). However, despite their higher enrolment percentages, which may paint a positive preliminary picture regarding women's experience in the academic system, particular indicators come into play to nuance this view. One of the most documented indicators regarding the gender issue in higher education is the variable of academic choices, generally in reference to the gender dynamics in STEM fields. In France (DEPP, 2018) as well as the majority of European Union countries (European Commission, 2012), women are still underrepresented in the fields of sciences, technology, engineering and mathematics. However, even when women enroll and persist in these fields, other features of the higher education environment may negatively interfere with their academic path. For instance, a study conducted in the United Kingdom on a population of chemistry doctoral students (Lober Newsome, 2008) revealed that women's interest in pursuing an 
academic career in the field decreased significantly during their doctoral studies, in part because of the competitive culture within the research teams. While in the first year, $72 \%$ of women and $61 \%$ of men expressed their interest in pursuing an academic career, by the third year, the percentage decreased to $37 \%$ among women compared to $59 \%$ among men. Moreover, there is often a smaller percentage of women than men among doctoral graduates even in domains in which they are in the majority at the undergraduate level. For example, according to a national French report (DEPP, 2018), 62\% of the bachelor's degree students enrolled in economics and administration were women. However, they represented only $42 \%$ of the students enrolled in the $\mathrm{PhD}$ programs in the same field. Research has also revealed that women experience higher belonging uncertainty (Harackiewicz et al., 2014; Harackiewicz, Canning, Tibbetts, Priniski, \& Hyde, 2015), including in fields where they are not targeted by a negative stereotype. These findings, which are illustrative rather than exhaustive, bring into question the idea that because they represent the majority of students, women would have an utterly positive psychological experience in higher education.

Previous research addressing women's poorer academic experience in higher education has focused on two main explanations: social identity threat (Major \& O'Brien, 2005) and the goals congruity hypothesis (Diekman, Clark, Johnston, Brown, \& Steinberg, 2011). The former occurs when women fear that they may be devalued in an academic setting because of their gender (e.g., Good, Rattan, \& Dweck, 2012; Murphy, Steele, \& Gross, 2007). The latter proposes that gendered endorsement of social goals leads to differentiated career paths, with women less likely to study in the most lucrative and prestigious STEM fields. It is worth noting the more recent approach of Leslie and colleagues (Leslie, Cimpian, Meyer, \& Freeland, 2015; Meyer, Cimpian, \& Leslie, 2015) that generalizes the deleterious effect of gender stereotypes beyond the STEM versus non-STEM framework. Their findings show that the percentage of women in a field of study may be predicted by the shared beliefs 
that success in the field requires brilliance, a feature stereotypically associated more with men (Bian, Leslie, Murphy, \& Cimpian, 2018; Verniers \& Martinot, 2015).

In the current research, we aim to go beyond these approaches, arguing that regardless of whether an academic setting is more or less congruent with women's goals or more or less targeted by negative stereotypes, the cultural marks associated with academic success in the increasingly selective realm of higher education may also negatively affect women's academic experience.

\section{A culture of selection in higher education}

Many Western European countries with minimal university entry requirements (e.g., France, Switzerland) have large first year enrollment and limit masters and doctoral students to those deemed competent or having merit (Darnon et al., 2009; Jury et al., 2016). This selection process creates a "struggle" to be perceived as competent, increasing students' willingness to demonstrate their own competences relative to others' (Jury et al., 2016). In the same vein, Stephens and colleagues (Stephens, Fryberg, Markus, Johnson, \& Covarrubias, 2012) illustrated that western higher educational institutions focus primarily on norms of independence (e.g., learn to be a leader, learn to influence others). The cultural frame created by these selection dynamics is theorized in Schwartz's model of values (for a review, Schwartz, 2012) as the self-enhancement values (e.g., ambition, competitiveness, self-affirmation, dominance). Self-enhancement corresponds to emphasizing competence in terms of prevailing social standards and the attainment of a dominant position within a social system. On the opposite side of the cultural spectrum, emphasizing the promotion of supportive social relations are the self-transcendence values (e.g., helpfulness, cooperation). This psychological opposition is well illustrated in the context of higher education. A low level of concern for others, often going as far as trying to impede others' progress, could be reasonably perceived as increasing the chances of success for oneself. For example, Toma 
and Butera (2009) showed that competition led group members to withhold unshared information in a decision-making task. To sum up, we argue that an academic culture that emphasizes self-enhancement values and deemphasizes self-transcendence values has become a pervasive feature of the current western academic system, regardless of whether a particular field is a STEM, non-STEM or brilliance-focused field. Because women endorse self-enhancement values less and self-transcendence values more than men do (Schwartz \& Rubel-Lifschitz, 2009; Schwartz \& Rubel, 2005), they may have a more challenging academic experience, notably in terms of belonging uncertainty, decreased academic selfefficacy, with downstream consequences on women's academic choices.

\section{Sense of belonging, academic self-efficacy and academic choices}

A sense of belonging in a given academic setting captures the extent to which students feel accepted, included, and supported by others (Goodenow, 1993). Students who experience a sense of belonging in educational environments are more engaged in classroom activities, more motivated, and report increased academic self-efficacy (Walton \& Cohen, 2007; Walton, Cohen, Cwir, \& Spencer, 2012). Academic self-efficacy captures students' belief that they are capable enough to master academic tasks and obtain successful results (Bandura, 1997). Thus, in the academic contexts in which women are less certain that they belong, they may consequently feel less academic self-efficacy. Furthermore, research has shown that academic self-efficacy in an educational field predicts students' course choices (Rudasill \& Callahan, 2010). However, so far, no research has tested such causal paths between these three psychological mechanisms (sense of belonging, academic self-efficacy and course choices) within the same study. Thus, the second goal of the present research is to examine within the same study how an academic culture promoting self-enhancement values may affect women's sense of belonging, self-efficacy, and course choices in both STEM and nonSTEM academic settings. 


\section{Overview of the research}

Past literature suggested that the culture often promoted in the STEM as well as brilliance-focused fields emphasizes self-enhancement values (competiveness, ambition, and dominance) (Hirshfield, 2010; Meyer et al., 2015). However, it remains unclear whether an individualistic and competitive culture would still have a negative effect outside a STEM or "brilliance-focused" type of setting. Indeed, in these settings self-enhancement values are embedded in an already threatening environment, with women being negatively stereotyped (Bian et al., 2018; Good et al., 2012; Murphy et al., 2007). Therefore, the goal of the present research is to isolate the effect of values alone on women's academic experience: first, within a STEM field but in a course not negatively stereotyped for women (Studies 1 and 2) and second, within a non-STEM field in a course framed as highly relevant and useful (Study 3). We argue that depicting self-enhancement values (versus self-transcendence values) as strongly linked to success in a given academic setting might undermine female students' expected sense of belonging, which would affect their anticipated self-efficacy, and ultimately would decrease the likelihood of integrating into the given academic setting, even if it is presented as highly relevant for their career. In contrast, due to the intrinsic nature of self-transcendence values, which foster a feeling of acceptance and integration for all students, both female and male students should report relatively high levels of sense of belonging, academic self-efficacy and interest in pursuing the presented academic opportunity in an academic culture depicting success in terms of self-transcendence values. Such a prediction mirrors the documented findings of men's positive experience in feminine occupations (Simpson, 2004). Thus, we hypothesized that assigning high success to selfenhancement values (as compared to self-transcendence values) would impair female students', but not male students', sense of belonging, academic self-efficacy and academic choices. 
Studies 1 and 2 tested this hypothesis on a population of engineering students. We considered that our hypothesis would receive stronger support if validated among women who had chosen a STEM field of study considered as especially prestigious and demanding in the French higher education system, hence embracing a career perceived as more favorable to self-enhancement than to self-transcendence values. Indeed, this will demonstrate that the negative effect of self-enhancement values remains strong even among women who chose such an orientation. We employed an experimental paradigm designed to minimize for women the potential social identity threat associated with the setting, by offering engineering students the opportunity to attend a psychology course. Moreover, in addition to lacking threatening STEM-related content, psychology is not perceived as a field in which brilliance is the primary requirement for success (Leslie et al., 2015).

In order to make the decision to participate to the course more consequential, and thus, increase the ecological validity of our research, Study 3 focused on psychology students. An institutional ethics committee approved the research protocol. The research materials and the data sets are available on Open Science Framework: https://osf.io/gsy48/?view_only=bc6b0f1583444b9abf4ed252bf971cf7

\section{Study 1}

\section{Method}

Participants. One hundred-fifteen third-year engineering students were randomly assigned either to the self-enhancement values condition (19 women and 37 men) or to the self-transcendence values condition (24 women and 35 men).

Procedure and materials. At the end of a regular class, the participants were told that their engineering school collaborated with the psychology department and that we were interested in their opinions on the potential implementation of a new psychology course the following academic year. After the course-description, participants either read that the 
students who succeed brilliantly in this course like being the ones making decisions, leading others, showing their competence and outperforming others (self-enhancement values condition) or that the students who succeed brilliantly in this course think it is important that all persons be treated equally, and like helping other students and listening to other people's different opinions even if they do not agree (self-transcendence values condition).

To ensure that the participants read and understood the text, they had to write down four adjectives that, in their opinion, characterized students who succeeded in this course. Next, they completed the 7-item sense of belonging scale (e.g., I could really be myself in this class, $1=$ not at all true to $5=$ completely true, $\alpha=.88$ adapted from Goodenow, 1993), and the 7-item academic self-efficacy scale (e.g., I'm certain I can master the skills taught in this class; $1=$ totally disagree to $7=$ totally agree; $\alpha=.91$, adapted from Midgley et al., 2000). Finally, the participants reported the likelihood of enrolling in this class the following academic year (i.e., "If the class were implemented the following academic year, would you enroll?"; 1 = not at all likely to $10=$ very likely).

\section{Results and Discussion}

\section{Manipulation check.}

Three blind judges estimated the extent to which each series of adjectives provided by the participants referred to self-enhancement values and self-transcendence values $(1=$ not at all; 7 = totally). The Cronbach's alpha coefficient showed good interrater reliability for both self-transcendence $(\alpha=.93)$ and self-enhancement $(\alpha=.84)$ ratings; therefore, the three judges' ratings were averaged to obtain a global score for self-transcendence $(M=4.54, S D=$ $2.22)$ and self-enhancement $(M=4.28, S D=1.84)$. Confirming that our manipulation worked, the adjectives provided in the self-enhancement values condition $(M=5.81, S E=$ 0.15) were indeed considered to refer to self-enhancement values to a significantly greater extent than those provided by the students in the self-transcendence values condition $(M=$ 
2.86, $S E=0.14), F(1,112)=204.48, p<.001$. Conversely, the adjectives provided in the self-transcendence values condition $(M=6.11, S E=0.20)$ were considered to refer to selftranscendence values to a significantly greater extent than the adjectives provided by the students in the self-enhancement values condition $(M=2.85, S E=0.20), F(1,112)=135.13$, $p<.001$.

\section{Overview of the analyses.}

We conducted regression analyses to test our hypotheses. The regression model contained three predictors: condition (coded -1 for the self-transcendence values condition and +1 for the self-enhancement values condition), gender (coded +1 for men and -1 for women), and condition $\mathrm{x}$ gender.

Sense of belonging. Regressing the sense of belonging scores on the predictors revealed a significant effect of condition, $B=-0.21,95 \%$ CI $[-0.37,-0.05], F(1,110)=6.60$, $p=.012, \mathrm{n}_{\mathrm{p}}{ }^{2}=.06$. When led to believe that success in the course was achieved through selfenhancement values $(M=2.96, S E=0.12)$, the participants expected a lower sense of belonging than when led to believe that succeeding involved the endorsement of selftranscendence values $(M=3.38, S E=0.11)$. The main effect of gender was not significant, $B$ $=0.10,95 \% \mathrm{CI}[-0.06,0.26], F(1,110)=1.40, p=.239, \mathrm{n}_{\mathrm{p}}^{2}=.01$. More importantly, the effect of condition was qualified by a significant condition $\mathrm{x}$ gender interaction effect, $B=$ $0.19,95 \%$ CI $[0.03,0.35], F(1,110)=5.37, p=.022, \eta_{\mathrm{p}}{ }^{2}=.05$ (Figure 1). Analyses of simple effects indicated that the women anticipated a significantly greater sense of belonging in the self-transcendence condition than in the self-enhancement condition, $B=-0.40,95 \%$ CI [$0.66,-0.14], F(1,110)=9.54, p=.003, \eta_{p}^{2}=.08$, whereas the men anticipated approximately the same sense of belonging across the two conditions, $F<1, p=.838$, $n s$. In the selfenhancement values condition, the women expected a significantly lesser sense of belonging than male students, $B=0.29,95 \% \mathrm{CI}[0.05,0.52], F(1,110)=5.80, p=.018, \mathrm{n}_{\mathrm{p}}{ }^{2}=.05$, 
whereas in the self-transcendence values condition, female and male students did not significantly differ regarding their anticipated sense of belonging, $F<1, p=.410$, ns.

Academic self-efficacy. Regressing the academic self-efficacy scores on the predictors revealed that neither the main effect of condition nor the main effect of gender was significant $(F<1, p=.382$, for condition; $F(1,110)=1.59, p=.209$, for gender). Although showing the expected means-pattern (Figure 2), the condition $\mathrm{x}$ gender interaction effect did not reach significance, $B=0.20,95 \% \mathrm{CI}[-0.04,0.45], F(1,110)=2.77, p=.099, \mathrm{n}_{\mathrm{p}}{ }^{2}=.03$.

Likelihood of enrolling in the course. Regressing the likelihood of enrolling scores on the predictors did not reveal any significant main or interaction effect ( $p s>.472, n s)$.

We found that in an experimentally created academic context, linking success to the endorsement of self-enhancement values (as opposed to self-transcendence values) undermined female students', but not male students', sense of belonging, creating a significant gender disparity. The effects expected on self-efficacy and likelihood of enrolling were not significant. One plausible explanation might be that the students' relatively busy timetable in engineering school lowered the general probability of choosing to attend a course that was neither essential nor mandatory for their training program. Furthermore, as we only had access to the students who attended the class that day, our sample sizes were quite small. To offset these limitations, we replicated Study 1 with the following methodological improvements. First, we used a-priori power analyses to determine the number of participants we needed to make sure we ran a sufficiently powered study. Second, we included in the description of the training more elements likely to appeal to engineering students (e.g., information encoding, human - machine interface). Since this modification in the content may cause the course to be seen as a "male" domain that may be threatening for women, we decided to assess students' perception of the course in terms of gender relevance. Third, because the one-item scale that we used to estimate the likelihood of enrolling in the 
course was quite limited in capturing participants' overall interest in the course, we improved the scale by including three more items.

\section{Study 2}

\section{Method}

Participants. We conducted first a power analysis using G*Power 3 (Faul, Erdfelder, Lang, \& Buchner, 2007) to determine how many participants we needed to achieve $80 \%$ power to detect a small to medium effect $\left(\eta_{p}^{2} \approx .04\right)$. This analysis suggested that we needed at least 191 participants. Two hundred thirty-four students from four French engineering schools completed the study. As detailed below, three participants were excluded due to manipulation check failure ${ }^{1}$. The final sample was of 231 participants $\left(M_{\mathrm{age}}=20.68, S D=\right.$ $1.89 ; 57$ women and 55 men in the self-enhancement values condition; 54 women and 65 men in the self-transcendence values condition).

Procedure and materials. Eighty-five students took part in the study at the end of a regular class and 146 completed the study on-line by accessing a Qualtrics link sent by their faculty $^{2}$. We used the same experimental paradigm as in Study 1, but with a more engineering-focused description of the training course, following which the students completed the sense of belonging $(\alpha=.86)$ and the academic self-efficacy $(\alpha=.88)$ scales. Next, the participant reported on their interest in enrolling in the course by answering four items (If the class were implemented the following academic year, I would enroll; I would like to participate to this training program; I find this training proposition particularly interesting; I think that I would not gain anything from this training course”, $1=$ not at all

${ }^{1}$ The results-pattern does not change when the three participants are included in the analyses.

${ }^{2}$ The results-pattern does not change when controlling for the study setting. 
likely, $10=$ very likely; $\alpha=.93)^{3}$. Finally, the participants rated on a 7-point scale whether the proposed training was intended more for women (response 1), for men (response 7) or both (response 4).

\section{Results and Discussion}

\section{Manipulation check.}

Two blind judges evaluated the adjective series. Three participants were excluded because their adjectives were unrelated to the actual description of the training. The interrater agreement was satisfactory for both self-transcendence $(r=.86, p<.001 ; M=2.96, S D$ $=2.12)$ and self-enhancement ratings $(r=.91, p<.001 ; M=2.23, S D=2.02)$. Supporting the efficacy of our manipulation, the adjectives provided in the self-enhancement values condition $(M=3.26, S E=0.17$ for self-enhancement; $M=1.64, S E=0.16$ for selftranscendence) were considered to refer to self-enhancement values to a greater extent, $F(1$, $215)=76.15, p<.001$, and to self-transcendence values to a lesser extent, $F(1,215)=$ $134.52, p<.001$, than those provided in the self-transcendence values condition $(M=1.20$, $S E=0.17$ for self-enhancement; $M=4.27, S E=0.16$ for self-transcendence).

\section{Perception of the training.}

A frequency analysis of this variable (Table 1) revealed that the vast majority of participants, namely, $89.6 \%$ of the total sample, considered that the training was intended for both men and women (i.e., response 4 on the scale), while $7.8 \%$ conveyed a rather gender neutral evaluation by choosing the responses 3 and 5 on the scale ${ }^{4}$.

${ }^{3}$ A factor analysis conducted on the four items revealed a single factor with an eigenvalue greater than 1 , accounting for $82 \%$ of the variance.

${ }^{4}$ The results-pattern does change when we excluded the five participants who assessed the course as being intended more for men (responses 5 and 6 on the scale). 
Overview of the analyses. As in Study 1, we contrast-coded our independent variables (condition: -1 for self-transcendence, +1 for self-enhancement; gender: +1 for men; -1 for women). Because students' age correlated with condition, standardized-age and its interactions with the independent variables were included in the final model (Yzerbyt, Muller, \& Judd, 2004) ${ }^{5}$. The regression model contained seven predictors: gender, condition, condition $\mathrm{x}$ gender, age, and the three interactions with age.

Sense of belonging. Regressing the sense of belonging scores on the predictors revealed a significant effect of condition, $B=-0.24,95 \% \mathrm{CI}[-0.34,-0.14], F(1,223)=23.71$, $p<.001, \mathrm{n}_{\mathrm{p}}^{2}=.10$. When led to believe that success in the course was achieved through selfenhancement values $(M=3.16, S E=0.07)$, the participants expected a lower sense of belonging than when led to believe that succeeding involved the endorsement of selftranscendence values $(M=3.64, S E=0.07)$. The main effect of gender was not significant, $B$ $=0.05,95 \% \mathrm{CI}[-0.05,0.15], F(1,223)=1.11, p=.292, \mathrm{\eta}_{\mathrm{p}}{ }^{2}=.01$. As expected, we obtained a significant condition $\mathrm{x}$ gender interaction effect, $B=0.12,95 \% \mathrm{CI}[0.02,0.22], F(1,223)=$ $5.71, p=.018, \mathrm{n}_{\mathrm{p}}{ }^{2}=.03$ (Figure 3). Analyses of simple effects indicated that the female students anticipated a significantly greater sense of belonging in the self-transcendence condition than in the self-enhancement condition, $B=-0.36,95 \% \mathrm{CI}[-0.50,-0.22], F(1,223)$ $=24.79, p<.001, \mathfrak{\eta}_{p}^{2}=.10$. The difference was not significant for the male students, $B=$ $0.12,95 \%$ CI $[-0.27,0.01], F(1,223)=3.28, p=.071, \eta_{p}^{2}=.01$. Furthermore, in the selfenhancement values condition, women expected a significantly lower sense of belonging than men did, $B=0.17,95 \%$ CI $[0.03,0.31], F(1,223)=5.63, p=.019, \eta_{p}{ }^{2}=.03$, whereas in the self-transcendence values condition, female and male students did not significantly differ regarding their anticipated sense of belonging, $F<1, p=.333$, $n s$. We also obtained a

\footnotetext{
${ }^{5}$ The results-pattern does not change when age was not entered into the model.
} 
significant interaction between age and condition, $B=-0.15,95 \% \mathrm{CI}[-0.27,-0.04], F(1,223)$ $=6.54, p=.011, \mathrm{n}_{\mathrm{p}}{ }^{2}=.03$. In the self-enhancement condition, the older the participants, the lower their expected sense of belonging, $B=-0.22,95 \% \mathrm{CI}[-0.41,-0.04], F(1,223)=5.61, p$ $=.019, \mathrm{n}_{\mathrm{p}}^{2}=.03$. In the self-transcendence condition, age was unrelated to sense of belonging $(p=.267)$

Academic self-efficacy. Regressing the academic self-efficacy scores on the predictors revealed main effects of condition and gender. When led to believe that success in the course was achieved through self-enhancement values $(M=4.51, S E=0.10)$, the participants anticipated lower academic self-efficacy than when led to believe that succeeding involved the endorsement of self-transcendence values $(M=4.87, S E=0.09), B=-0.18,95 \%$ CI $[-0.32,-0.04], F(1,223)=6.79, p=.010, \eta_{\mathrm{p}}{ }^{2}=.03$. Overall, the male students $(M=3.83$, $S E=0.09)$ anticipated higher academic self-efficacy than the female students $(M=4.55, S E=$ $0.10), B=0.14,95 \%$ CI $[0.004,0.28], F(1,223)=4.09, p=.044, \eta_{p}{ }^{2}=.02$. The condition $\mathrm{x}$ gender interaction effect was significant, $B=0.21,95 \%$ CI $[0.07,0.34], F(1,223)=8.99, p=$ $.003, \mathrm{n}_{\mathrm{p}}{ }^{2}=.04$ (Figure 4). Analyses of the simple effects indicated that the female students anticipated higher academic self-efficacy in the self-transcendence condition than in the selfenhancement condition, $B=-0.39,95 \% \mathrm{CI}[-0.58,-0.19], F(1,223)=14.79, p<.001 . \mathrm{n}_{\mathrm{p}}{ }^{2}=$ .06. The male students anticipated comparably the same academic self-efficacy across the two conditions $(F<1, p=.775, n s)$. Moreover, in the self-enhancement values condition, the women expected lower academic self-efficacy than men did, $B=0.35,95 \%$ CI $[0.15,0.54]$, $F(1,223)=11.95, p=.001, \mathrm{n}_{\mathrm{p}}^{2}=.05$, whereas in the self-transcendence values condition, female and male students did not significantly differ regarding their expected academic selfefficacy, $F<1, p=.479, n s$. Finally, although the interaction between age and condition was significant, $B=-0.19,95 \%$ CI $[-0.35,-0.02], F(1,223)=5.14, p=.024, \eta_{\mathrm{p}}^{2}=.02$, the simple effects of age did not reach significance ( $p=.119$ in the self-enhancement condition; $p=.093$ 
in the self-transcendence condition).

Likelihood of enrolling in the course. Regressing the likelihood of enrolling scores on the predictors revealed a significant effect of condition, $B=-0.54,95 \% \mathrm{CI}[-0.83,-0.25]$, $F(1,223)=13.28, p<.001, \mathrm{n}_{\mathrm{p}}^{2}=.06$. When led to believe that success in the course was achieved through self-enhancement values $(M=6.15, S E=0.21)$, the participants reported a lower interest in the course than when led to believe that succeeding involved the endorsement of self-transcendence values $(M=7.22, S E=0.20)$. The main effect of gender was not significant, $F<1, p=.531, n s$. We obtained a significant condition $\mathrm{x}$ gender interaction effect, $B=0.48,95 \% \mathrm{CI}[0.19,0.77], F(1,223)=10.78, p=.001, \mathrm{n}_{\mathrm{p}}^{2}=.05$ (Figure 5). Analyses of simple effects indicated that the female students reported a greater interest in the course in the self-transcendence condition than in the self-enhancement condition, $B=-1.02,95 \%$ CI $[-1.44,-0.60], F(1,223)=22.59, p<.001, \eta_{\mathrm{p}}{ }^{2}=.09$, whereas the male students reported similar interest in the course across the two conditions, $F<1, p=$ $.792, n s$. In the self-transcendence values condition, women expressed a greater interest in the course than men did, $B=-0.58,95 \%$ CI $[-0.97,-0.18], F(1,223)=8.08, p=.005, \mathrm{n}_{\mathrm{p}}{ }^{2}=$ .04 . This difference was not significant in the self-enhancement values condition, $B=0.39$, $95 \%$ CI $[-0.03,0.81], F(1,223)=3.35, p=.069, \eta_{p}^{2}=.02$. A significant interaction between age and condition was revealed, $B=-0.54,95 \%$ CI $[-0.88,-0.19], F(1,223)=9.25, p=.003$, $\eta_{\mathrm{p}}{ }^{2}=.04$. In the self-enhancement condition, the older the participants, the lower their interest in the course, $B=-0.76,95 \%$ CI $[-1.31,-0.21], F(1,223)=7.36, p=.007, \mathrm{n}_{\mathrm{p}}{ }^{2}=.03$. In the self-transcendence condition, age was unrelated to likelihood of enrolling ( $p=.146)$.

Mediation analyses. To test the hypothesized mediated moderation model (Figure 6), we used the PROCESS macro for SPSS (Model 6; 5000 bootstrap samples; Hayes, 2018), in which we entered the interaction term between condition $\mathrm{x}$ gender as the $\mathrm{X}$ variable, and the main effects of gender and condition in the covariates window. Age and its interactions with 
the independent variables were also included as covariates. The interaction effects of condition $\mathrm{x}$ gender on likelihood of enrolling ( $\mathrm{c}$ path) and sense of belonging ( $\mathrm{a}_{1}$ path) have already been established as significant. The effect of sense of belonging on academic selfefficacy, when controlling the condition $\mathrm{x}$ gender interaction effect ( $\mathrm{d}_{21}$ path), was also significant, $B=0.61,95 \%$ CI $[0.45,0.78], t(222)=7.29, p<.001$. Finally, the effect of academic self-efficacy on likelihood of enrolling, when controlling the effects of sense of belonging and condition $\mathrm{x}$ gender interaction ( $\mathrm{b}_{2}$ path), was significant, $B=0.51,95 \% \mathrm{CI}$ $[0.25,0.77], t(221)=3.91, p<.001$, whereas the interaction effect between condition $\mathrm{x}$ gender on likelihood of enrolling (c' path) was not, $B=0.23,95 \%$ CI $[-0.02,0.47], t(221)=$ $1.83, p=.068$. Moreover, the indirect effect of condition $\mathrm{x}$ gender on likelihood of enrolling through the sense of belonging and academic self-efficacy in the sequence (path $\mathrm{a}_{1} * \mathrm{~d}_{21} * \mathrm{~b}_{2}$ ) was significant, $B=0.04,95 \%$ CI $[0.01,0.09]$.

Taken together, Studies 1 and 2 consistently demonstrated that depicting selfenhancement values as strongly linked to success undermined female students', but not male students', sense of belonging. Study 2 went further in showing that such an association also undermined female students' academic self-efficacy, and interest in the course. Importantly, the fact that the course proposed to the participants was a psychology course, a field that is not negatively stereotyped for women and which was not perceived as a male domain, allowed for an independent test of the effect of values. Finally, we obtained an interesting result involving students' age. In an academic culture depicting self-enhancement values as highly linked to success, the older the students the lower their anticipated sense of belonging and their interest in the course. This may be explained by the fact that the older the students, the more they have been exposed to such a culture, generating more avoidance tendencies. Although this effect applies to both male and female students, it does so in an additive manner for women only, because, independent of age, they are affected more by the self- 
enhancement culture compared to men.

We argued that similar selection processes should apply to all fields of study. Thus, even a "social” field of study (e.g., psychology) could develop a culture of achievement based on self-enhancement values, and thus create a challenging academic context for female students. Therefore, the goal of Study 3 was to test the generalizability of the findings among psychology students and to highlight further the academic relevance of the findings. Moreover, the results of Studies 1 and 2 illustrated that, as expected, male students were unaffected by whether the achievement culture in the course emphasized self-transcendence or self-enhancement values. Thus, we conducted Study 3 on female psychology students and framed the psychology course as highly important for their career.

\section{Study 3}

\section{Method}

Participants. Based on a medium effect size $\left(\eta_{p}{ }^{2} \approx .08\right)$, as suggested by the effect sizes obtained in Study 2 for women, a G*Power 3 analysis (Faul et al., 2007) indicated that we needed at least 93 participants to achieve $80 \%$ power. Ninety-seven first and second year female psychology students completed the study (43 in the self-enhancement values condition; 54 in the self-transcendence values condition).

Procedure and materials. We used the same experimental paradigm and framed the psychology course as highly relevant for today's job market. Next, students completed the sense of belonging scale $(\alpha=.89)$, academic self-efficacy scale $(\alpha=.91)$, and estimated their likelihood of enrolling in this class the following academic year $(1=$ not at all likely to $10=$ very likely). In order to reinforce the perception of an actual academic choice they were about to make, the students were asked to report their name and academic identification number.

\section{Results and Discussion}

Manipulation check. Three blind judges evaluated the four adjective series with 
satisfying inter-rater agreement for both self-transcendence $(\alpha=.93, M=4.36, S D=2.28)$ and self-enhancement ratings $(\alpha=.77, M=4.35, S D=1.81)$. Confirming that our manipulation worked, the adjectives provided in the self-enhancement values condition $(M=$ $5.96, S E=0.17)$ were indeed considered to refer to self-enhancement values to a significantly greater extent than those provided by the students in the self-transcendence values condition $(M=3.07, S E=0.15), F(1,95)=167.78, p<.001$. Conversely, the adjectives provided in the self-transcendence values condition $(M=5.98, S E=0.19)$ were considered to refer to selftranscendence values to a significantly greater extent than the adjectives provided by the students in the self-enhancement values condition $(M=2.33, S E=0.21), F(1,95)=167.82, p$ $<.001$.

Sense of belonging. As in previous studies, we regressed the scores for sense of belonging on condition ( -1 for the self-transcendence values condition; +1 for the selfenhancement values condition). When led to believe that success in the course was achieved through self-enhancement values $(M=2.83, S E=0.13)$, the participants expected a lower sense of belonging than when led to believe that succeeding implied endorsing selftranscendence values $(M=3.53, S E=0.11), B=-0.35,95 \%$ CI $[-0.52,-0.18], F(1,95)=$ $16.64, p<.001, \mathrm{n}_{\mathrm{p}}^{2}=.15$.

Academic self-efficacy. Regressing the scores for academic self-efficacy on condition revealed that when led to believe that success in the course was achieved through selfenhancement values $(M=4.05, S E=0.16)$, the participants anticipated a lower level of academic self-efficacy than when led to believe that succeeding implied endorsing selftranscendence values $(M=4.83, S E=0.15), B=-0.39,95 \% \mathrm{CI}[-0.60,-0.17], F(1,95)=$ $12.60, p=.001, \mathrm{n}_{\mathrm{p}}^{2}=.12$..

Likelihood of enrolling in the course. Regressing the scores of likelihood of enrolling in the course on condition revealed that when led to believe that success in the 
course was achieved through self-enhancement values $(M=3.93, S E=0.35)$, the participants were less likely to enroll in the course than when led to believe that succeeding implied endorsing self-transcendence values $(M=5.98, S E=0.32), B=-1.02,95 \%$ CI $[-1.50,-0.56]$, $F(1,94)=18.78, p<.001, \mathrm{n}_{\mathrm{p}}^{2}=.17$

\section{Mediation analyses: What model best captures the path from condition to}

\section{likelihood of enrolling?}

We hypothesized that that a culture of achievement based on self-enhancement values ultimately affects female students' academic choices through its effect on students' anticipated sense of belonging and academic self-efficacy. However, given our cross sectional data, we could not exclude the alternative, that the path from condition to likelihood of enrolling for the course flows through anticipated academic self-efficacy and expected sense of belonging (i.e., Model 2). Thus, in order to assess which of the two models better fits our data, we conducted path analyses using a maximum likelihood (ML) estimator in R. To test our hypothesis (i.e., Model 1), we ran a model with estimated paths from condition to sense of belonging, from sense of belonging to academic self-efficacy and from academic self-efficacy to likelihood of enrolling in the course. Moreover, to show full mediation, we constrained the direct path from condition to likelihood of enrolling in the course to zero. Additionally, to depict full meditation of the effect of condition on academic self-efficacy through sense of belonging, we also constrained the direct path from condition to academic self-efficacy to zero. Based on previous studies documenting a direct effect of sense of belonging on academic choices (e.g., Good et al., 2012), we allowed for the direct path from sense of belonging to likelihood of enrolling to be estimated. Supporting our hypothesis, this model showed good fit to the data, $\chi^{2}(2)=4.09, p=.130, C F I=.99, R M S E A=.10,90 \% \mathrm{CI}$ 
$[.00, .25]^{6}$, and $S R M R=.05$ (Figure 7 ). To test the conceptual alternative model (i.e., Model 2), we shifted the place of sense of belonging with academic self-efficacy. As in Model 1, to reflect full mediation, the direct path from condition to likelihood of enrolling was set to zero. Moreover, to mirror Model 1, we set the direct path from condition to sense of belonging to zero, while allowing for the direct path from academic self-efficacy to likelihood of enrolling to be estimated ${ }^{7}$. Model 2 showed poor fit to the data, with a statistically significant $\chi^{2}$ test, $\chi^{2}(2)=7.66, p=.022$.

These complementary sets of analyses support the directionality of our hypothesized model. Depicting self-enhancement values (as opposed to self-transcendence values) as strongly linked to success undermined female students' sense of belonging, academic selfefficacy, and corresponding choice regarding enrolling in the course. As the course was framed as a clearly useful and relevant opportunity for their future career, this result effectively illustrates the dynamic through which, even in a non-STEM field of study, women opt out of academic possibilities, with a potentially decisive impact on their professional career, when they perceive that they have to endorse self-enhancement values to succeed.

${ }^{6}$ It is important to note that the RMSEA tends to be substantially elevated for correctly specified models with small degree of freedom (Kenny, Kaniskan, \& Mccoach, 2015). In fact, the authors recommend completely avoiding computing the RMSEA when model dfs are small, and particularly when the $\chi^{2}$ test is not statistically significant, since, in such a case, we know that the model relatively closely reproduces the data.

${ }^{7}$ Given that in the alternative model we did not have a specific prediction about the degree of mediation for the effect of condition on sense of belonging through academic selfefficacy, we also ran Model 2b, in which we included a direct path from condition to sense of belonging. Model $2 \mathrm{~b}$ also showed relatively poor fit to the data, $\chi^{2}(1)=2.98, p=.084$. 


\section{General Discussion}

Women's academic attainment in higher education is qualified by a certain paradox. Despite the fact that a higher proportion of women than men attend university, women's psychological experience in various academic contexts is qualified by decreased sense of belonging, and academic self-efficacy, even in non-STEM settings, contradicting interpretations in terms of social identify threat only (e.g., Harackiewicz et al., 2015; Sommet et al., 2013). We proposed that a possible explanation may be located at a cultural level, namely in the emergence, within an increasingly selective higher education system, of an achievement culture based on self-enhancement values.

Because of gender differences in the endorsement of self-enhancement values (Schwartz \& Rubel-Lifschitz, 2009; Schwartz \& Rubel, 2005), we expected that an academic setting depicting success in terms of self-enhancement values (as opposed to selftranscendence values) would diminish female students' sense of belonging, academic selfefficacy and interest in pursuing the given academic course. Indeed, we showed that independent of fields, STEM (Studies 1 and 2) or non-STEM (Study 3), an academic culture depicting self-enhancement values as highly linked to success had a negative impact on female students' psychological academic experience through sense of belonging (the three studies), self-efficacy and academic choices (Studies 2 and 3). This is important because even a "social" field of study, in which the specific goals afforded by the future career (e.g., being a psychologist, hence helping people), may be close to self-transcendence values, could still develop a culture of achievement based on self-enhancement values. This may be the case because similar selection processes apply to all fields of study, STEM or social sciences (Darnon et al., 2009). Moreover, by presenting female engineering students with a course that does not pose a particular threat to their gender identity, we assured that the effect is due to the value-mismatch. Thus, our findings nicely disentangled the effect of values from the 
effect of social identity threat, which are relatively intertwined in the literature. Furthermore, we extended the goals congruency hypothesis (Diekman et al., 2011) by highlighting a complementary academic dynamic in which the concept of values is essential, but which is orthogonal to the students' primary choices for specific fields of study. In other words, both students who chose either a STEM-related or a female-dominated field of study may be confronted during their studies with a culture of success emphasizing self-enhancement values. Understanding how students navigate this climate is of particular importance. Thus, an additional contribution of the present results is to depict the psychological mechanism underlying students' academic choices. Based on the literature (Rudasill \& Callahan, 2010; Walton et al., 2012), we construed a mediational process engaging as key variables sense of belonging and academic self-efficacy. Although the cross-sectional nature of our data demands caution, the various statistical techniques employed support the idea of sense of belonging affecting academic self-efficacy, which ultimately shapes students' interest in different academic courses. The effect on self-efficacy, which was modeled as fully mediated by sense of belonging, is particularly interesting since it reveals that women's belief in their capacity to master academic tasks and obtain successful results is affected by cultural features of the setting unrelated to the actual content of the course.

\section{Limitations and perspectives}

In the current research, we argue that self-enhancement values are associated with a high level of academic success, notably because of the selection function inherent in the functioning of the higher education system. However, we have not fully addressed the direct causal relationship between the selection practices of the university and the perception of the academic culture as being based on self-enhancement values. For instance, showing that the link between self-enhancement values and academic success is stronger when the type of evaluation in the course is based on relative performance as opposed to personal 
improvement (Smeding, Darnon, Souchal, Toczek-Capelle, \& Butera, 2013) or in fields practicing numerus clausus (Sommet et al., 2013) would nicely test this relationship.

Despite these limitations, our findings have significant practical implications. In particular, given the selection processes that take place in most European academic systems (e.g., France, Belgium, Switzerland) all throughout the undergraduate level, an academic culture in which self-enhancement values are emphasized is rather common, even in a nonSTEM field of study. For example, the fact that a program is "highly competitive" is often presented as an argument to support its quality. Such messages imply a display of selfenhancement values and, as suggested by our findings, could prevent female students from taking full advantage of their academic opportunities, even in a field stereotypically in accordance with their predominant values and goals. This is particularly important because it suggests that all things being equal, women may have a more challenging academic experience, even if they perform as well as their male counterparts (e.g., Harackiewicz et al., 2014, 2015). Women's generally good performance in college may minimize and even conceal their more subjective experience in terms of sense of belonging or self-efficacy, with significant downstream consequences for their academic choices during their academic years. Finally, in the spirit of self-transcendence values, higher educational institutions could certainly gain from implementing collaborative projects framed with a prosocial purpose. For example, the opportunity of working together toward common ends was found to increase motivation for challenging tasks, as well as greater interest in and enjoyment of the task (Butler \& Walton, 2013; Carr \& Walton, 2014). Our results suggest that such collaborative projects might also provide all students with a more positive academic experience. 


\section{References}

Bandura, A. (1997). Self-efficacy: The exercise of control. New York: W.H. Freeman.

Bian, L., Leslie, S. J., Murphy, M. C., \& Cimpian, A. (2018). Messages about brilliance undermine women's interest in educational and professional opportunities. Journal of Experimental Social Psychology, 76(January), 404-420.

https://doi.org/10.1016/j.jesp.2017.11.006

Butler, L. P., \& Walton, G. M. (2013). The opportunity to collaborate increases preschoolers' motivation for challenging tasks. Journal of Experimental Child Psychology, 116(4), 953-961. https://doi.org/10.1016/j.jecp.2013.06.007

Carr, P. B., \& Walton, G. M. (2014). Cues of working together fuel intrinsic motivation. Journal of Experimental Social Psychology, 53, 169-184. https://doi.org/10.1016/j.jesp.2014.03.015

Darnon, C., Dompnier, B., Delmas, F., Pulfrey, C., \& Butera, F. (2009). Achievement goal promotion at university: social desirability and social utility of mastery and performance goals. Journal of Personality and Social Psychology, 96(1), 119-134.

https://doi.org/10.1037/a0012824

Diekman, A. B., Clark, E. K., Johnston, A. M., Brown, E. R., \& Steinberg, M. (2011). Malleability in communal goals and beliefs influences attraction to STEM careers: Evidence for a goal congruity perspective. Journal of Personality and Social Psychology, 101(5), 902-918. https://doi.org/10.1037/a0025199

European Commission. (2012). Gender in Research and Innovation. Brussels.

Faul, F., Erdfelder, E., Lang, A. G., \& Buchner, A. (2007). G*Power 3: A flexible statistical power analysis program for the social, behavioral, and biomedical sciences. Behavior Research Methods, 39(2), 175-191. https://doi.org/10.3758/BF03193146

Fiske, E. B., \& UNESCO. (2012). Atlas mondial de l'égalité des genres dans l'éducation. 
Paris: HarperCollins.

Good, C., Rattan, A., \& Dweck, C. S. (2012). Why do women opt out? Sense of belonging and women's representation in mathematics. Journal of Personality and Social Psychology, 102(4), 700-717. https://doi.org/10.1037/a0026659

Goodenow, C. (1993). The psychological sense of school membership among adolescents: Scale development and educational correlates. Psychology in the Schools, 30, 79-90. https://doi.org/10.1177/095968369100100211

Harackiewicz, J. M., Canning, E. A., Tibbetts, Y., Giffen, C. J., Blair, S. S., Rouse, D. I., \& Hyde, J. S. (2014). Closing the social class achievement gap for first-generation students in undergraduate biology. Journal of Educational Psychology, 106(2), 375-389. https://doi.org/10.1037/a0034679

Harackiewicz, J. M., Canning, E. A., Tibbetts, Y., Priniski, S. J., \& Hyde, J. S. (2015). Closing achievement gaps with a utility-value intervention: Disentangling race and social class. Journal of Personality and Social Psychology, in press, No Pagination Specified. https://doi.org/10.1037/pspp0000075

Hirshfield, L. E. (2010). "She Won’t Make Me Feel Dumb": Identity Threat in a MaleDominated Discipline. International Journal of Gender, Science and Technology, 2(1), $6-24$.

Jury, M., Darnon, C., Dompnier, B., \& Butera, F. (2016). The social utility of performanceapproach goals in a selective educational environment. Social Psychology of Education. https://doi.org/10.1007/s11218-016-9354-

Kenny, D. A., Kaniskan, B., \& Mccoach, D. B. (2015). The Performance of RMSEA in Models With Small Degrees of Freedom. https://doi.org/10.1177/0049124114543236

Leslie, S.-J., Cimpian, A., Meyer, M., \& Freeland, E. (2015). Expectations of brilliance underlie gender distributions across academic disciplines. Science, 347(6219), 23-34. 
https://doi.org/10.1081/E-EWS

Lober Newsome, J. (2008). The chemistry PhD : the impact on women's retention. London. Major, B., \& O’Brien, L. T. (2005). The social psychology of stigma. Annual Review of Psychology, (56), 393-421. https://doi.org/10.1146/annurev.psych.56.091103.070137

Meyer, M., Cimpian, A., \& Leslie, S. J. (2015). Women are underrepresented in fields where success is believed to require brilliance. Frontiers in Psychology, 6(MAR), 1-12. https://doi.org/10.3389/fpsyg.2015.00235

Midgley, C., Maehr, M. L., Hruda, L. Z., Anderman, E., Anderman, L., Freeman, K. E., ... Urdan, T. (2000). Patterns of Adaptive Learning Scales. University of Michigan.

Murphy, M. C., Steele, C. M., \& Gross, J. J. (2007). Signaling Threat. How Situational Cues Affect Women in Math, Science, and Engineering Settings. Psychological Science, 18(10), 879-885. https://doi.org/10.1111/j.1467-9280.2007.01995.x

Rudasill, K. M., \& Callahan, C. M. (2010). Academic Self-Perceptions of Ability and Course Planning Among Academically Advanced Students. Journal of Advanced Academics, 21(2), 300-329. https://doi.org/10.1177/1932202X1002100206

Schwartz, S. H. (2012). An overview of the Schwartz theory of basic values. Online Readings in Psychology and Culture, 2(1), 1-20.

https://doi.org/http://dx.doi.org/http://dx.doi.org/10.9707/2307-0919.1116

Schwartz, S. H., \& Rubel-Lifschitz, T. (2009). Cross-national variation in the size of sex differences in values: effects of gender equality. Journal of Personality and Social Psychology, 97(1), 171-185. https://doi.org/10.1037/a0015546

Schwartz, S. H., \& Rubel, T. (2005). Sex differences in value priorities: cross-cultural and multimethod studies. Journal of Personality and Social Psychology, 89(6), 1010-1028. https://doi.org/10.1037/0022-3514.89.6.1010

Smeding, A., Darnon, C., Souchal, C., Toczek-Capelle, M. C., \& Butera, F. (2013). Reducing 
the socio-economic status achievement gap at University by promoting mastery-oriented assessment. PLoS ONE, 8(8), 1-6. https://doi.org/10.1371/journal.pone.0071678

Sommet, N., Pulfrey, C., \& Butera, F. (2013). Did my M.D. really go to University to learn? Detrimental effects of numerus clausus on self-efficacy, mastery goals and learning. PLoS ONE, 8(12), 1-15. https://doi.org/10.1371/journal.pone.0084178

Stephens, N. M., Fryberg, S. A., Markus, H. R., Johnson, C. S., \& Covarrubias, R. (2012). Unseen Disadvantage : How American Universities ' Focus on Independence Undermines the Academic Performance of First-Generation College Students, 102(6), 1178-1197. https://doi.org/10.1037/a0027143

Toma, C., \& Butera, F. (2009). Hidden profiles and concealed information: Strategic information sharing and use in group decision making. Personality and Social Psychology Bulletin, 35(6), 793-806. https://doi.org/10.1177/0146167209333176

Verniers, C., \& Martinot, D. (2015). Characteristics expected in fields of higher education and gender stereotypical traits related to academic success: a mirror effect. Social Psychology of Education, 11218. https://doi.org/10.1007/s11218-015-9312-z

Walton, G. M., \& Cohen, G. L. (2007). A question of belonging: race, social fit, and achievement. Journal of Personality and Social Psychology, 92(1), 82-96. https://doi.org/10.1037/0022-3514.92.1.82

Walton, G. M., Cohen, G. L., Cwir, D., \& Spencer, S. J. (2012). Mere belonging: The power of social connections. Journal of Personality and Social Psychology, 102(3), 513-532. https://doi.org/10.1037/a0025731

Yzerbyt, V. Y., Muller, D., \& Judd, C. M. (2004). Adjusting researchers Õ approach to adjustment: On the use of covariates when testing interactions q, 40, 424-431. https://doi.org/10.1016/j.jesp.2003.10.001 